\title{
Crystal Structure of SAV0927 and Its Functional Implications
}

\author{
Soyeon Jeong ${ }^{1}$, Hyo Jung $\mathrm{Kim}^{2}$, Nam-Chul $\mathrm{Ha}^{1 *}$, and Ae-Ran Kwon ${ }^{3 *}$ \\ ${ }^{1}$ Department of Agricultural Biotechnology, Center for Food Safety and Toxicology, Center for Food and Bioconvergence, Research Institute \\ for Agriculture and Life Sciences, Seoul National University, Seoul 08826, Republic of Korea \\ ${ }^{2}$ Centre for Biomolecular Sciences, School of Pharmacy, University of Nottingham, Nottingham NG7 2RD, UK \\ ${ }^{3}$ Department of Herbal Skin Care, College of Herbal Bioindustry, Daegu Haany University, Gyeongsan 38610, Republic of Korea
}

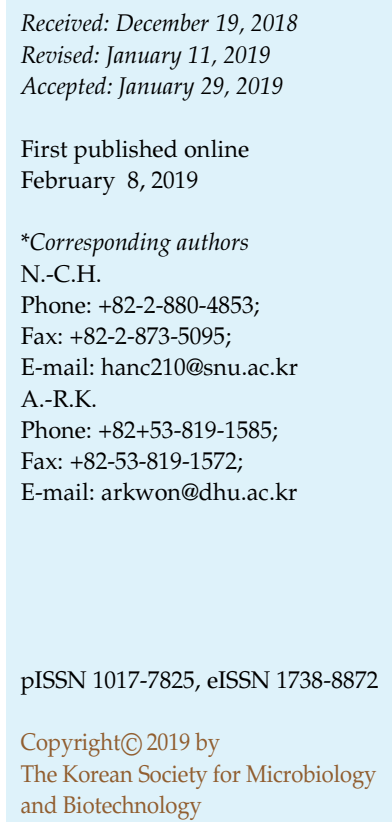

Staphylococcus aureus is a round-shaped, gram-positive bacterium that can cause numerous infectious diseases ranging from mild infections such as skin infections and food poisoning to life-threatening infections such as sepsis, endocarditis and toxic shock syndrome. Various antibiotic-resistant strains of $S$. aureus have frequently emerged, threatening human lives significantly. Despite much research on the genetics of $S$. aureus, many of its genes remain unknown functionally and structurally. To counteract its toxins and to prevent the antibiotic resistance of $S$. aureus, our understanding of $S$. aureus should be increased the proteomic scale. SAV0927 was first sequenced in an antibiotic resistant $S$. aureus strain. The gene is a conserved hypothetical protein, and its homologues appear to be restricted to Firmicutes. In this study, we determined the crystal structure of SAV0927 at $2.5 \AA$ resolution. The protein was primarily dimeric both in solution and in the crystals. The asymmetric unit contained five dimers that are stacked linearly with $\sim 80^{\circ}$ rotation by each dimer, and these interactions further continued in the crystal packing, resulting in a long linear polymer. The crystal structures, together with the network analysis, provide functional implications for the SAV0927-mediated protein network.

Keywords: SAV0927, DUF3055, Staphylococcus aureus, MRSA, X-ray crystallography

\section{Introduction}

Staphylococcus aureus is a round-shaped, gram-positive bacterium that is frequently found as a commensal in humans. However, it is one of the major causes of community- and hospital-acquired infections [1]. It causes all sorts of infectious diseases ranging from mild infections, such as skin infections and food poisoning, to lifethreatening infections, such as sepsis, endocarditis and toxic shock syndrome [2]. However, the prognosis for patients with $S$. aureus infection is often poor despite early diagnosis and appropriate treatments because $S$. aureus frequently acquires antibiotic resistance [3]. Very little is known on how $S$. aureus acquires antibiotic resistance at the molecular level despite extensive studies. In this regard, understanding the bacterial defense mechanisms used by $S$. aureus against antibiotics is crucial.
Whole genome sequencing of many antibiotic-resistant $S$. aureus strains has been performed to understand its antibiotic resistance as well as the pathogenicity of the bacterium $[1,4,5]$. SAV0927 was primarily sequenced in $S$. aureus strain Mu50 (ATCC 700699), which is an MRSA strain with additional vancomycin-intermediate-resistance [1]. SAV0927 is a relatively small protein consisting of 88 amino acids without a signal sequence for secretion. It was annotated as a conserved hypothetical protein mostly constituted by a DUF3055 domain whose structure is not known yet.

This gene is also found in other species of the Staphylococcus genus, including S. haemolyticus, and S. edaphicus, as well as other strains of S. aureus (locus WP_001637673) with almost $100 \%$ identity in the matched region. It appears to be restricted to Firmicutes, and no structure is available to date. To gain insight into the function of SAV0927, we 
determined the crystal structure of the hypothetical protein at a high resolution in this study. Based on the structure, we proposed a possible function of SAV0927.

\section{Materials and Methods}

\section{Construction, Protein Expression and Purification of SAV0927}

The gene coding for SAV0927 (NCBI reference sequence: BA000017.4) was amplified from the genomic DNA from S. aureus Mu50 by using PCR. The restriction enzymes NdeI and XhoI were used to insert the PCR products into these two sites in a pET21a(+) vector, resulting in pET21a(+)-SAV0927, which contains a hexahistidine tag at the C-terminus of the protein for purification. For the production of the selenomethionine (SeMet)-labeled protein, SAV0927 was expressed in the E. coli B834 (DE3) strain, which was cultured in $3 \mathrm{~L}$ of $\mathrm{M} 9$ medium in the presence of Lselenomethionine at $37^{\circ} \mathrm{C}$ until the $\mathrm{OD}_{600}$ reached 0.8 [6]. The SAV0927 protein expression was induced with $0.5 \mathrm{mM}$ IPTG at $30^{\circ} \mathrm{C}$ for $6 \mathrm{~h}$. After cell harvest by centrifugation, the cell pellet was resuspended in $50 \mathrm{ml}$ of lysis buffer containing $20 \mathrm{mM}$ Tris $\cdot \mathrm{HCl}$ ( $\mathrm{pH} 8.0$ ), $150 \mathrm{mM} \mathrm{NaCl}$, and $2 \mathrm{mM} \beta$-mercaptoethanol. The cells were disrupted by sonication, and the cell debris was removed by centrifugation at $20,000 \mathrm{~g}$ for $30 \mathrm{~min}$ at $4^{\circ} \mathrm{C}$. The hexahistidine tagged protein was purified using Ni-NTA agarose resin ( $2 \mathrm{ml}$; Qiagen, Germany). The protein-bound resin was washed with $300 \mathrm{ml}$ of a buffer containing $20 \mathrm{mM}$ Tris $\cdot \mathrm{HCl}(\mathrm{pH}$

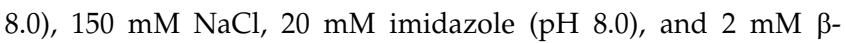
mercaptoethanol, and then the protein was subsequently eluted with $30 \mathrm{ml}$ of a buffer containing $20 \mathrm{mM}$ Tris $\mathrm{HCl}(\mathrm{pH} 8.0), 150 \mathrm{mM}$ $\mathrm{NaCl}, 250 \mathrm{mM}$ imidazole ( $\mathrm{pH} 8.0$ ), and $2 \mathrm{mM} \beta$-mercaptoethanol. The protein was further purified with an anion exchange chromatographic column by applying a gradient from $0 \mathrm{mM}$ to $1 \mathrm{M} \mathrm{NaCl}$. The protein was eluted in a range of $250-450 \mathrm{mM} \mathrm{NaCl}$. After concentration to $5 \mathrm{ml}$ using a Vivaspin centrifugal concentrator (10 kDa molecular-weight cutoff; Millipore), a gel filtration chromatographic column (HiLoad 16/60 Superdex 200; GE Healthcare) was used in $20 \mathrm{mM}$ Tris $\cdot \mathrm{HCl}$ buffer $(\mathrm{pH} 8.0)$ containing $150 \mathrm{mM} \mathrm{NaCl}$, and $2 \mathrm{mM} \beta$-mercaptoethanol. The pooled fractions were concentrated to $19 \mathrm{mg} / \mathrm{ml}$ using the same concentrator and stored frozen at $-80^{\circ} \mathrm{C}$ until use.

\section{Crystallization}

Initial crystallization trials of the SAV0927 protein were performed using an automated crystal screening device (Mosquito) at $14^{\circ} \mathrm{C}$ with the sitting-drop vapor-diffusion method. Five matrix screening solutions (MCSG-1T/ 2T/3T/4T, Anatrace and HR-110/112, Hampton Research Crystal Screen) that are commercially available were selected. The protein solution $(0.2 \mu \mathrm{l}, 19 \mathrm{mg} / \mathrm{ml})$ was mixed with a reservoir solution $(0.2 \mu \mathrm{l})$ and equilibrated against $60 \mu \mathrm{l}$ of the reservoir solution in a 96-well crystallization plate. Plateshaped crystals were obtained under the reservoir solution containing $0.1 \mathrm{M}$ Tris $\cdot \mathrm{HCl}(\mathrm{pH} 8.5)$ and $1.5 \mathrm{M}$ ammonium sulfate.
Table 1. X-ray data statistics and refinement of the structure.

\begin{tabular}{|c|c|}
\hline & SAV0927 \\
\hline \multicolumn{2}{|l|}{ Data collection } \\
\hline Beam line & PAL7A \\
\hline Wavelength $(\AA)$ & 0.97934 \\
\hline Space group & $P 2_{1}$ \\
\hline \multicolumn{2}{|l|}{ Cell dimensions } \\
\hline a, b, c $(\AA)$ & $128.2,76.8,43.6$ \\
\hline$\alpha, \beta, \gamma\left({ }^{\circ}\right)$ & $90,90.4,90$ \\
\hline Resolution $(\AA)$ & $50.0-2.50(2.54-2.50)$ \\
\hline $\mathrm{R}_{\text {merge }}$ & $0.081(0.397)$ \\
\hline $\mathrm{R}_{\mathrm{pim}}$ & $0.034(0.174)$ \\
\hline$I / \sigma I$ & $18.9(6.7)$ \\
\hline Completeness (\%) & $99.3(99.3)$ \\
\hline Redundancy & $6.6(6.0)$ \\
\hline \multicolumn{2}{|l|}{ Refinement } \\
\hline Resolution (Å) & $28.8-2.49$ \\
\hline No. reflections & 29255 \\
\hline $\mathrm{R}_{\text {work }} / \mathrm{R}_{\text {free }}$ & $0.237 / 0.278$ \\
\hline No. of Total atoms & 5933 \\
\hline Wilson B-factor $(\AA)$ & 24.9 \\
\hline \multicolumn{2}{|l|}{ R.M.S deviations } \\
\hline Bond lengths $(\AA)$ & 0.003 \\
\hline Bond angles $\left(^{\circ}\right)$ & 0.518 \\
\hline \multicolumn{2}{|l|}{ Ramachandran plot } \\
\hline Favored (\%) & 98.7 \\
\hline Allowed (\%) & 1.3 \\
\hline Outliers (\%) & 0.0 \\
\hline PDB code & $6 \mathrm{IY} 0$ \\
\hline
\end{tabular}

The crystallization conditions were optimized with the hangingdrop diffusion method under a reservoir solution containing $0.1 \mathrm{M}$ Tris $\cdot \mathrm{HCl}(\mathrm{pH} 7.5)$ and $1.4 \mathrm{M}$ ammonium sulfate at $14^{\circ} \mathrm{C}$. In the final optimization experiments, larger rod-shaped crystals suitable for data collection in 2 weeks were obtained in a 15-well plate by mixing $1 \mu \mathrm{l}$ of the protein solution $(19 \mathrm{mg} / \mathrm{ml})$ and $1 \mu \mathrm{l}$ of the reservoir solution in $500 \mu \mathrm{l}$ of the reservoir solution.

\section{Structural Determination}

For data collection under cryogenic conditions, the crystals were incubated for $90 \mathrm{~min}$ in cryo-protection buffer containing $0.05 \mathrm{M}$ Tris $\mathrm{HCl}(\mathrm{pH} 7.5)$ and saturated ammonium sulfate. The crystals were flash-cooled in liquid nitrogen at $-173^{\circ} \mathrm{C}$. The datasets were collected on an ADSC Quantum Q270 CCD detector in beamline 7A of Pohang Accelerator Laboratory, Republic of Korea [7], at a wavelength of $0.97934 \AA$. The program HKL-2000 was used to process the diffraction datasets [8]. The structure of 
SAV0927 was determined and refined using Coot and Phenix [9, 10]. Table 1 describes the data collection and refinement of structure statistics.

\section{Results}

\section{Protein Expression and Structural Determination of SAV0927}

The gene encoding SAV0927 was amplified by PCR using S. aureus Mu50 genomic DNA and was inserted into an expression vector containing a hexahistidine tag. The fulllength SAV0929 was overexpressed in E. coli cells and purified to homogeneity with $>95 \%$ purity (Fig. 1). The SEC-MALS result showed the SAV0927 protein was $\sim 22 \mathrm{kDa}$ in solution, which indicates a dimer is the fundamental oligomeric state of this protein (Fig. 1). The crystals were obtained at $0.1 \mathrm{M}$ Tris. $\mathrm{HCl}(\mathrm{pH} 7.5)$ and $1.4 \mathrm{M}$ ammonium sulfate and the diffraction data were collected at $2.5 \AA$ resolution in the space group of $P 2_{1}$. The electron density map from the Se-Met substituted crystal by the SAD phasing was sufficient to trace the entire protein chains (Table 1).

\section{Dimeric Structure of SAV0927}

Five dimers were contained in the asymmetric units (Fig. 2A). However, one dimer from the five dimers was mostly disordered except for the central dimeric interface.

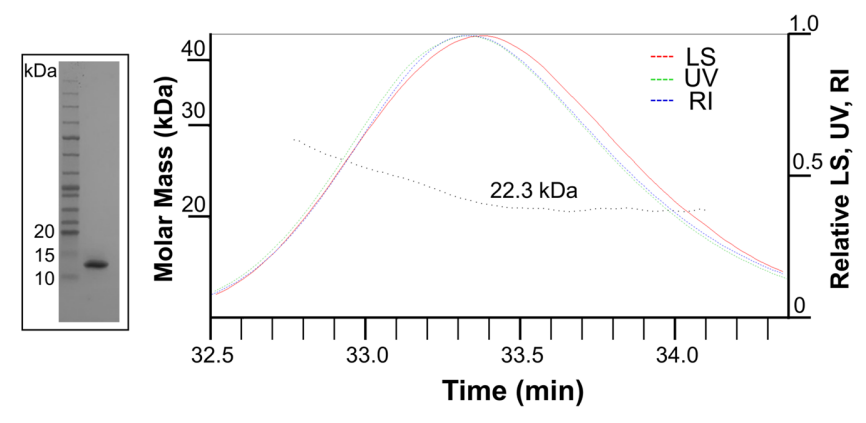

Fig. 1. The purified SAV0927 protein.

Left, the purified protein was subjected to SDS-PAGE using a 4-20\% gradient gel to analyze the purity and the molecular weight. Right, the purified protein was further analyzed by SEC-MALS using Superdex 200 Increase 10/300 GL (GE Healthcare). The left axis shows the molar mass and the right axis shows the relative protein concentrations that were evaluated by light scattering (LS), ultraviolet (UV) at $280 \mathrm{~nm}$, and refractive index (RI).

The two protomers in each dimer were tightly interacting, consistent with the SEC-MALS result (Figs. 2A and 1). Each protomer contains an anti-parallel five-stranded $\beta$-sheet, one of which is from the other subunit in the dimer $\left(\beta 1^{\prime}\right)$. The first $\beta$-strand $(\beta 1)$ is the longest and the $N$-terminal half part is involved in the swapped $\beta$-strands between the protomers in the dimer. The last $\beta$-strand $(\beta 4)$ continued to
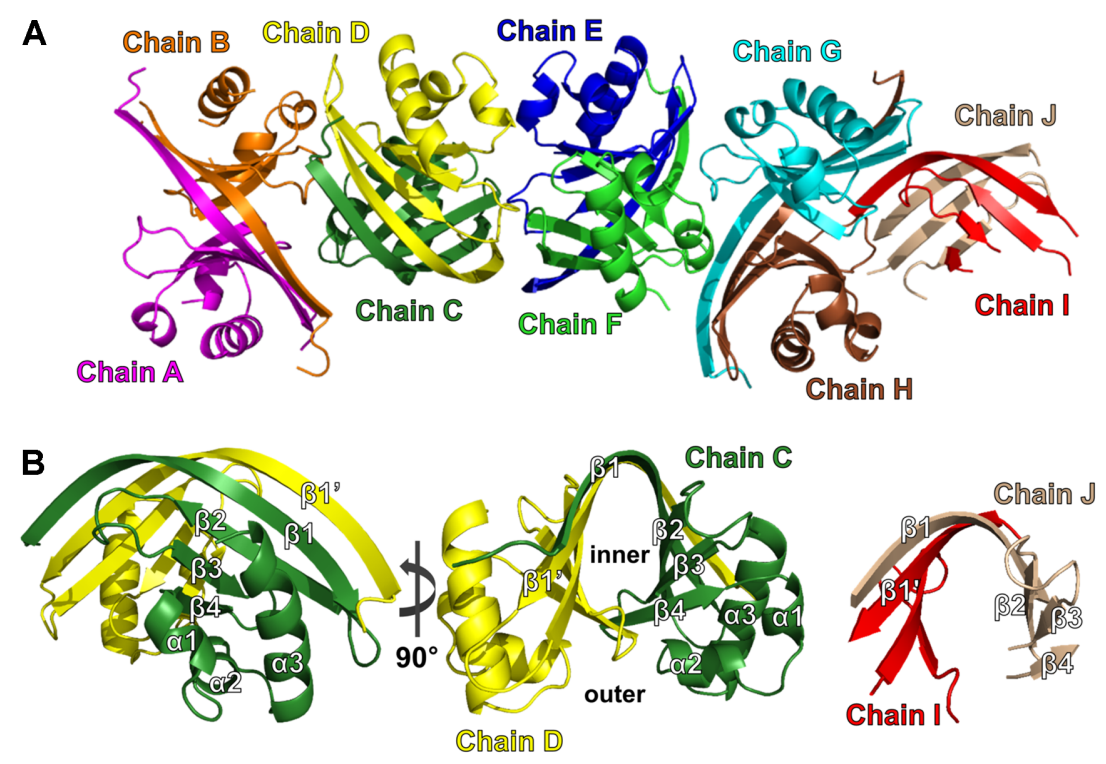

Fig. 2. Structure of the asymmetric unit.

(A) Five dimers (A:B, C:D, E:F, G:H, I:J) in the asymmetric unit are displayed in the cartoon representation. Each protomer is colored differently. (B) The dimeric unit of SAV0927. Each secondary structure in a protomer (dark green) is labeled. $\beta 1^{\prime}$ from the other protomer (yellow) is labeled to show the swapped $\beta$-strands between the protomers. The top view of the barrel of the dimer is displayed in the central panel. In the right panel, the chains I and J are displayed (red and wheat), whose $\alpha$-helical parts are disordered. 
the three $\alpha$-helices at the C-terminus. Of these, the Cterminal end $\alpha$-helix $(\alpha 3)$ is the longest and one portion is exposed on the surface. The two $\beta$-sheets from the dimer make a $\beta$-barrel in the centric dimeric (inner) face, and the other (outer) face of the $\beta$-sheet makes another hydrophobic interaction with the hydrophobic face of the C-terminal $\alpha 3$ helix (Fig. 2B). In the fifth dimer (chain I and chain J) in the asymmetric unit, only the central $\beta$-barrel region is ordered, and the $\alpha$-helices are not ordered, indicating the structural rigidity of the central $\beta$-barrel in the dimer (Fig. 2B).

The protein fold-matching program DALI server [11] of the protomer did not suggest any structural homologues that have a similar $\beta$-barrel of the dimer, which is described below. These findings suggest that SAV0927 has a novel fold or structural organization.

\section{DUF3055 Domain-Containing Proteins}

All of the DUF3055-containing proteins have a high sequence similarity in their DUF3055 domain. The entire amino acid sequence of DUF3055 domain is the same as that of SAV0927. However, an additional 7-11 residues were found in other species of Staphylococcus and other Firmicutes (Fig. 3A). The C-terminal end of SAV0927 in the $\alpha$-helix is near the interface between the two dimers (Fig. 3B). It is unlikely that the divergent C-terminal tail sequence plays an important role in the function of SAV0927.

\section{A Linear Polymer Formation in the Crystal}

Each dimer is stacked linearly along the axis with $\sim 28 \AA$ length and $\sim 80^{\circ}$ rotation (Fig. $4 \mathrm{~A}$ ). Thus, five dimers in the asymmetric unit make a cylinder with a $60 \AA$ diameter and a $140 \AA$ height. Especially, His37 and Tyr38 from the loop connecting $\beta 2$ and $\beta 3$ form the main interface for this interaction (Fig. 4B). His37 and Tyr38 make polar interactions with Tyr38 and His37 from the adjacent dimer, respectively. Apparent pockets are found in every interface between the dimers (see red arrow in Fig. 4A). Five residues (Arg36, His37, Tyr38, Lys40, and Ile53) comprise the hydrophobic pocket, whose size appears to accommodate a molecule as large as ATP $(\mathrm{Mw}=503)$ with $13 \AA$ in the length and $8 \AA$ in the width (Fig. 4C).

These interactions further and infinitively continue to the neighboring dimeric units in the crystal packing (see blue arrows in Fig. 4A), resulting in the formation of a very long linear polymer (Fig. 4A). The appearance of the linear polymer with pockets at the interface between the dimeric
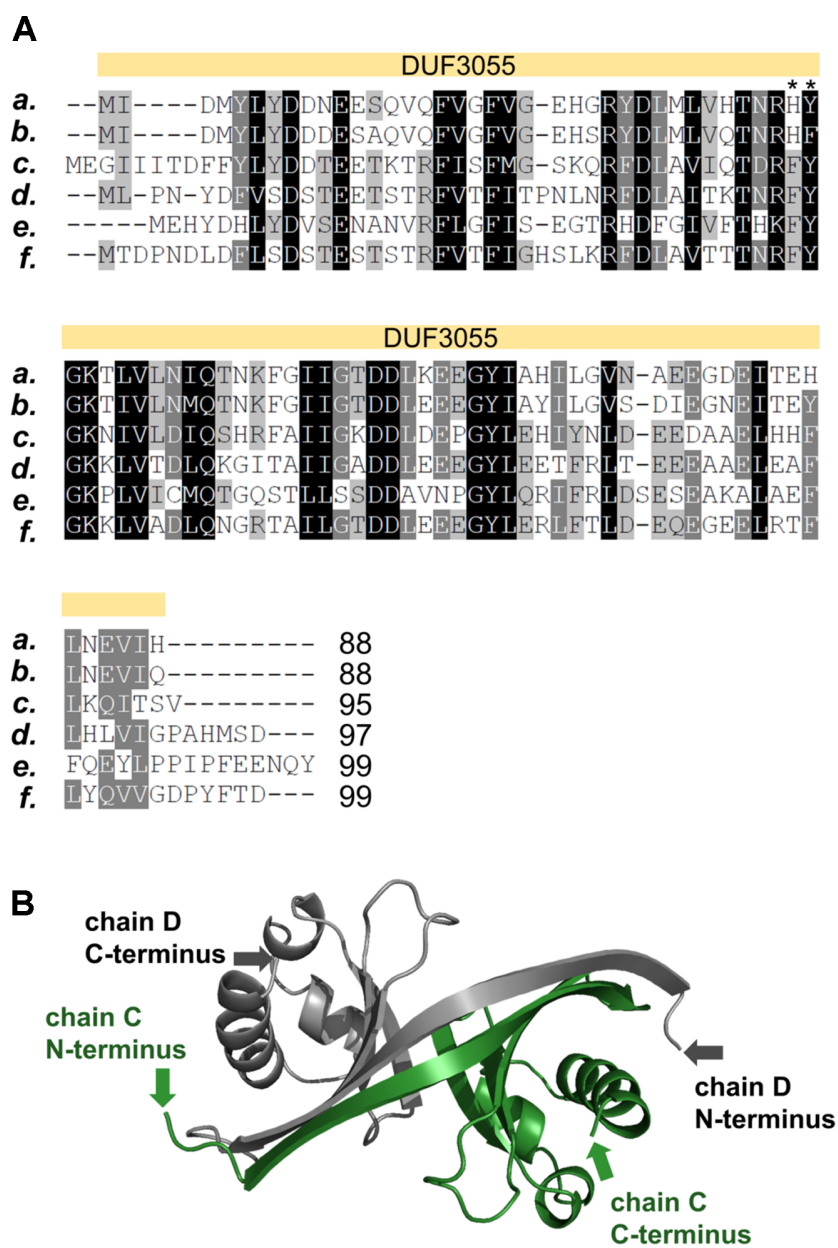

Fig. 3. Sequence alignment of DUF3055-containing proteins and dimer structure of SAV0927.

(A) Conserved amino acid sequences are highlighted. The DUF3055 domain is indicated above the sequences. $a$ for DUF3055 containing protein (SAV0927) from Staphylococcus aureus; b from Staphylococcus edaphicus; $c$ from Bacillus azotoformans MEV2011; $d$ from Thermobacillus composti KWC4; e from Brevibacillus sp. CF112; $f$ from Paenibacillus aceti. The asterisk marks above the amino acids indicate the residues involved in interaction between the adjacent dimers (see Fig. 4B). (B) $\mathrm{N}$-terminus and $\mathrm{C}$-terminus are represented respectively at chain $\mathrm{C}$ (green arrow) and chain D (grey arrow) of SAV0927.

unit tempted us to speculate on the existence of ligands to the pockets that augment the linear polymer formation found in the crystal packing.

\section{Discussion}

To gain functional insights, we used the STRING database, which is a bioinformatics tool that presents known and 
A

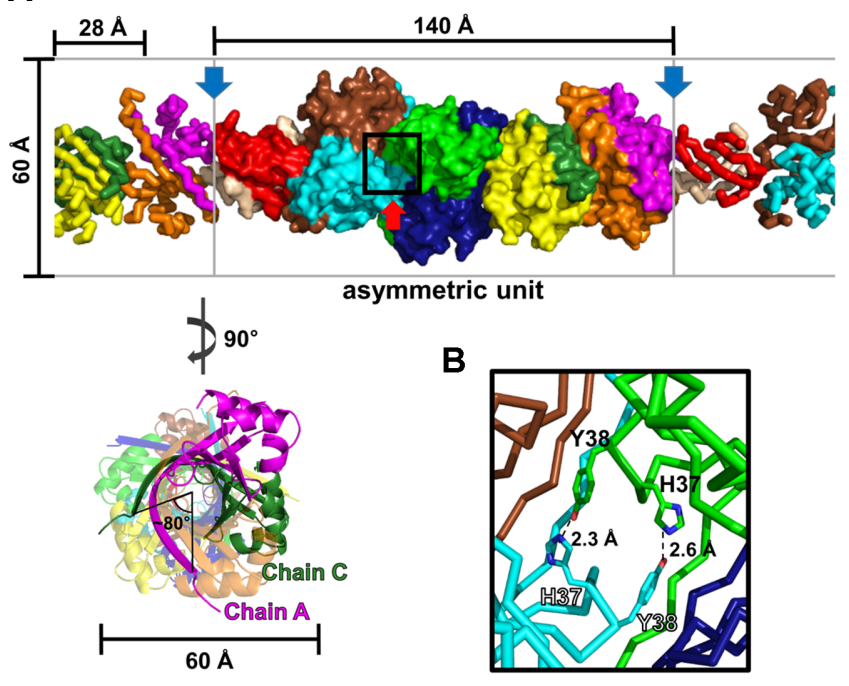

C

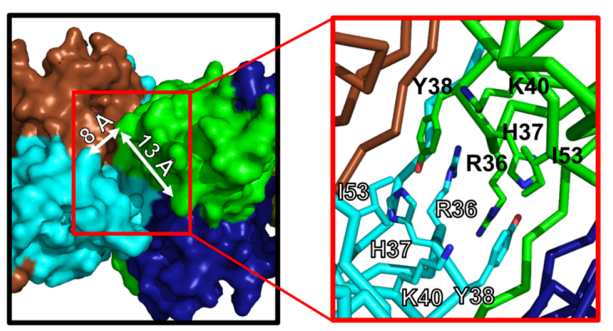

Fig. 4. Linearly arranged SAV0927 in the crystal.

Each gray box represents the asymmetric unit, and the two side boxes are truncated. (A) The packing interactions between the asymmetric units are indicated by blue arrows. Top view of the asymmetric unit is shown at the bottom left. One of the interactions between the dimers are boxed and enlarged at the bottom right. Each interface between the dimers has a pocket indicated by a red arrow. (B) This inset is magnified in the black box at Fig. 4A. His37 and Tyr38 residues are shown at stick and polar interactions and appear at the dotted line. (C) The left panel of the black box is a magnified view of the hydrophobic pocket at Fig. 4A. The white arrows indicate the length and width of the pocket. The red boxed region in the left panel is enlarged at the right panel. Five residues in the hydrophobic pocket are displayed in the stick representations (right panel).

predicted protein-protein interactions [12]. The STRING predicted that SAV0927 might be functionally related to SAV0928, SAV1496, SAV1289, SAV1290, SAV1015, NagD, SAV1430, SAV0939, FtsL, and SAV1877, although the function of none of these has been confirmed experimentally (Fig. 5). All of these proteins are hypothetical and functionally uncharacterized except NagD and FtsL. NagD is in its neighborhood, and FtsL showed a gene co-occurrence with SAV0927.

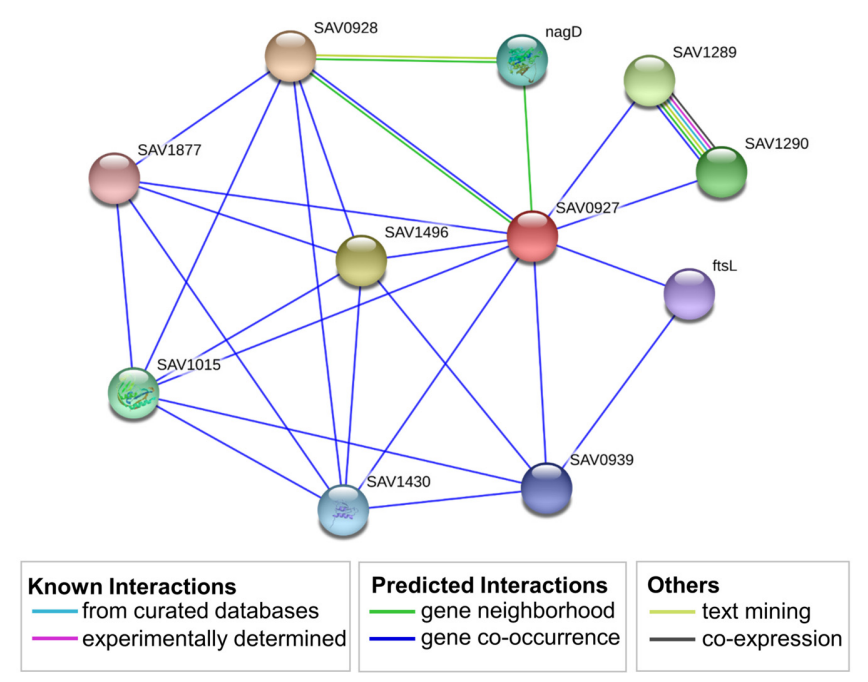

Fig. 5. STRING analysis focusing on SAV0927.

The STRING database version was 10.5 (http:/ / string-db.org).

$\mathrm{NagD}$ is an N-acetyl-glucosamine catabolism-like protein that catalyzes dephosphorylation of 2-6 carbon acid sugars [13]. E. coli NagD specifically dephosphorylates nucleoside monophosphate with two conserved core domains. The dimeric SAV0927 structure does not contain a noticeable pocket or the possible catalytic residues. Thus, it would not be likely that SAV0927 exhibits an enzymatic activity linked to the activity of $\mathrm{NagD}$ at least in its dimeric form.

It might be noteworthy that it has a possible functional relationship to FtsL, which plays a central role in the initiation of cell division, probably by transducing a signal for activation of peptidoglycan remodeling at the forming septum [14]. An $\alpha$-helix of FtsL was proposed to act as a potential mechanical connection to communicate conformational changes between the periplasmic, membrane, and cytoplasmic regions in E. coli and Bacillus species [14]. Furthermore, FtsL was characterized to form a protein complex with DvlB and DvlC [15]. The key protein for the septum remodeling is tubulin-like FtsZ that forms a linear polymer with a treadmilling action using nucleotides [16]. FtsZ is the first protein recruited to the division site and is functionally related to the FtsL-DvlB-DvlC complex in S. aureus [16].

The linear polymeric forms of SAV0927 observed in the crystal structure provide interesting functional insights. The noticeable pockets were found between the dimers in the linear polymer, which might be involved in the enzymatic actions related to NagD. Alternatively, it is also possible that inter-linear polymer interactions between SAV0927 and FtsZ (or FtsZ complex) would be augmented 
in the linear polymeric forms. However, it is obvious that further studies on SAV0927 are required to reveal the function of SAV0927. To investigate the function of SAV0927, the linear polymeric form should be considered as a functional form.

S. aureus is one of the most important human pathogens and has been extensively studied. To prevent the virulence and the antibiotic resistance of this bacteria, understanding of the biology of $S$. aureus at the proteomic scale might be required. This study will be helpful in understanding the full features of $S$. aureus.

\section{Acknowledgments}

This research was supported by the Korea Institute of Planning and Evaluation for Technology in Food, Agriculture, Forestry (IPET) through the Agriculture, Food and Rural Affairs Research Center Support Program, funded by the Ministry of Agriculture, Food and Rural Affairs (MAFRA) (710012-03-1-HD120 to NCH). This work was also supported through the National Research Foundation of Korea (NRF) funded by the Ministry of Education of the Korean government (2010-0025488 and 2017R1D1A1B03033857 to ARK). We made use of beamlines 7A at Pohang Accelerator Laboratory (Pohang, Republic of Korea).

\section{Conflict of Interest}

The authors have no financial conflicts of interest to declare.

\section{References}

1. Kuroda M, Ohta T, Uchiyama I, Baba T, Yuzawa $H$, Kobayashi I, et al. 2001. Whole genome sequencing of meticillin-resistant Staphylococcus aureus. Lancet 357: 1225-1240.

2. Archer GL. 1998. Staphylococcus aureus: a well-armed pathogen. Clin. Infect. Dis. 26: 1179-1181.

3. Woods C, Colice G. 2014. Methicillin-resistant Staphylococcus aureus pneumonia in adults. Expert. Rev. Respir. Med. 8: 641-651.

4. Harris SR, Cartwright EJ, Torok ME, Holden MT, Brown NM, Ogilvy-Stuart AL, et al. 2013. Whole-genome sequencing for analysis of an outbreak of meticillin-resistant Staphylococcus aureus: a descriptive study. Lancet Infect. Dis. 13: 130-136.

5. Humphreys H, Coleman DC. 2013. Whole genome sequencing and the prevention and control of meticillin-resistant Staphylococcus aureus infection. J. Hosp. Infect. 85: 85-86.

6. Guerrero SA, Hecht HJ, Hofmann B, Biebl H, Singh M. 2001. Production of selenomethionine-labelled proteins using simplified culture conditions and generally applicable host/ vector systems. Appl. Microbiol. Biotechnol. 56: 718-723.

7. Park S, Ha S, Kim Y. 2017. The protein crystallography beamlines at the Pohang light source II. Biodesign 5: 30-34.

8. Otwinowski Z, Minor W. 1997. Processing of X-ray diffraction data collected in oscillation mode. Methods. Enzymol. 276: 307-326.

9. Emsley P, Cowtan K. 2004. Coot: model-building tools for molecular graphics. Acta Crystallogr. D, Biol. Crystallogr. 60: 2126-2132.

10. Adams PD, Afonine PV, Bunkoczi G, Chen VB, Davis IW, Echols N, et al. 2010. PHENIX: a comprehensive Pythonbased system for macromolecular structure solution. Acta Crystallogr. D, Biol. Crystallogr. 66: 213-221.

11. Holm L, Laakso LM. 2016. Dali server update. Nucleic Acids Res. 44: W351-355.

12. Szklarczyk D, Morris JH, Cook H, Kuhn M, Wyder S, Simonovic M, et al. 2017. The STRING database in 2017: quality-controlled protein-protein association networks, made broadly accessible. Nucleic Acids Res. 45: D362-D368.

13. Tremblay LW, Dunaway-Mariano D, Allen KN. 2006. Structure and activity analyses of Escherichia coli K-12 NagD provide insight into the evolution of biochemical function in the haloalkanoic acid dehalogenase superfamily. Biochemistry 45: 1183-1193.

14. Condon SGF, Mahbuba DA, Armstrong CR, Diaz-Vazquez G, Craven SJ, LaPointe LM, et al. 2018. The FtsLB subcomplex of the bacterial divisome is a tetramer with an uninterrupted FtsL helix linking the transmembrane and periplasmic regions. J. Biol. Chem. 293: 1623-1641.

15. Sievers J, Errington J. 2000. The Bacillus subtilis cell division protein FtsL localizes to sites of septation and interacts with DivIC. Mol. Microbiol. 36: 846-855.

16. Monteiro JM, Pereira AR, Reichmann NT, Saraiva BM, Fernandes PB, Veiga H, et al. 2018. Peptidoglycan synthesis drives an FtsZ-treadmilling-independent step of cytokinesis. Nature 554: 528-532. 